\section{Pre-surgical medical treatment, a major prognostic factor of remission in acromegaly.}

\author{
F.Albarel ${ }^{*}$, F.Castinetti*, I.Morange ${ }^{*}$, B.Conte-Devolx ${ }^{*}$, N. Guibert**, H.Dufour***, \\ T.Brue*.
}

Department of endocrinology*, statistics ** and neurosurgery***, Timone hospital, 264 rue St Pierre, 13005 Marseille, France.

Following the recent evolution in therapeutic strategies for GH-secreting pituitary adenomas, determining optimal individualized patient management is now crucial.

Objective: To determine whether pre-surgical medical treatment (PSMT) in patients with acromegaly improves surgical outcome and to specify thresholds for such a strategy.

Methods and design: This retrospective study included 110 newly diagnosed acromegalic patients operated on between 1997 and 2007 at Timone Hospital, Marseille, France. The mean long-term follow-up period was $52 \pm 36.6$ months (median 41 months). Sixty-four patients (58.4\%) received PSMT (long acting Somatostatin Analogs) during 2 to 18 months (mean 6.4 months) and all patients underwent pituitary surgery. Remission was based on updated criteria, associating GH nadir after oral glucose tolerance test $<0.4 \mu \mathrm{g} / \mathrm{L}$ and normal IGF-1 for age, sex and gender at early (3 months) evaluation or at the end of follow-up ( $\mathrm{n}=95)$.

\title{
Results:
}

\begin{abstract}
At 3 months and at long-term evaluation, pre-treated and no pre-treated groups were comparable for the main confounding factors except for IGF1 at diagnosis which was higher in patients with PSMT at both evaluations.
\end{abstract}

3 months Evaluation

Data

\begin{tabular}{|c|c|c|c|}
\hline Data & $\begin{array}{c}\text { Patients with } \\
\text { PSMT }(6.4+/-4.1) \\
5 \text { months median } \\
n=64 \\
\end{array}$ & $\begin{array}{c}\text { Patients no } \\
\text { PSMT } \\
\text { n=46 }\end{array}$ & $\mathbf{p}$ \\
\hline $\begin{array}{l}\text { - Age at diagnosis (years) } \\
\text { - GHm at diagnosis }(\mu \mathrm{g} / \mathrm{L})\end{array}$ & $\begin{array}{l}44.1 \pm 10.7 \\
31.4 \pm 35.8\end{array}$ & $\begin{array}{l}45 \pm 14.6 \\
30.1 \pm 38.6\end{array}$ & $\begin{array}{l}0.7 \\
0.87\end{array}$ \\
\hline - IGF-1 at diagnosis (\% ULN) & $3.5 \pm 1.4$ & $2.9 \pm 1.5$ & 0.04 \\
\hline - GH nadir after OGTT $(\mu \mathrm{g} / \mathrm{L})$ & $26 \pm 32.2$ & $25.7 \pm 33.6$ & 0.90 \\
\hline - Pre-surgical adenoma size $(\mathrm{mm})$ & $16.6 \pm 6.5$ & $18.6 \pm 13.3$ & 0.32 \\
\hline - Macro/micro adenoma (\% patients) & 82.6 & 81.2 & $>0.9$ \\
\hline - Local Invasion (\% of patients) & 54.7 & 54.4 & $>0.9$ \\
\hline - Visual Field abnormalities (\% patients) & 26 & 27 & $>0.9$ \\
\hline - Hyperprolactinemia (\% of patients) & 31.2 & 30.4 & $>0.9$ \\
\hline - Pre-surgical pituitary deficiency (\% patients) & 48.4 & 39.1 & 0.44 \\
\hline - Age at surgery (years) & $44.9 \pm 10.8$ & $45.2 \pm 14.2$ & 0.89 \\
\hline - Delay before surgery (years) & $0.9 \pm 0.5$ & $0.6 \pm 0.9$ & 0.03 \\
\hline - endoscopic surgery (\% patients) & 21.9 & 17.4 & 0.63 \\
\hline - Impression of complete tumor removal (\% patients) & 46.9 & 34.8 & 0.47 \\
\hline
\end{tabular}

In multivariate analysis, PSMT was significantly linked to:

. Early remission: $45.3 \%$ patients in remission with PSMT vs $26.1 \%$ without; [(OR=3.17 (1.15-8.73), $\mathbf{p = 0 . 0 0 9}$.

.Long-term remission: $61.1 \%$ patients in remission with PSMT vs $36.6 \%$ without; [(OR=2.74 (1.04-7.26), $\mathbf{p = 0 . 0 2 2}]$.

\section{Long-Term Evaluation}

\begin{tabular}{|c|c|c|c|}
\hline Data & $\begin{array}{c}\text { Patients with } \\
\text { PSMT (6.5 +/- 4.1) } \\
5 \text { months median } \\
\mathbf{n}=54\end{array}$ & $\begin{array}{c}\text { Patients no } \\
\text { PSMT } \\
\text { n=41 }\end{array}$ & $\mathbf{p}$ \\
\hline - Age at diagnosis (years) & $44 \pm 10.7$ & $44 \pm 14.3$ & $>0.9$ \\
\hline - GHm at diagnosis $(\mu \mathrm{g} / \mathrm{L})$ & $33.3 \pm 36.6$ & $31.6 \pm 40.8$ & 0.84 \\
\hline - IGF-1 at diagnosis (\% ULN) & $3.4 \pm 1.3$ & $2.8 \pm 1.5$ & 0.03 \\
\hline - GH nadir after OGTT $(\mu \mathrm{g} / \mathrm{L})$ & $27.3 \pm 33.6$ & $26.4 \pm 35.3$ & 0.92 \\
\hline - Pre-surgical adenoma size $(\mathrm{mm})$ & $16.9 \pm 6.9$ & $19.1 \pm 13.9$ & 0.33 \\
\hline - Macro/micro adenoma (\% patients) & 77.8 & 80.5 & 0.8 \\
\hline - Local Invasion (\% of patients) & 59.3 & 53.7 & 0.68 \\
\hline - Visual Field abnormalities (\% patients) & 28.9 & 27.3 & $>0.9$ \\
\hline - Hyperprolactinemia (\% of patients) & 31.5 & 29.3 & $>0.9$ \\
\hline - Pre-surgical pituitary deficiency (\% patients) & 48.1 & 39 & 0.41 \\
\hline - Age at surgery (years) & $44.9 \pm 10.8$ & $44.3 \pm 14$ & 0.82 \\
\hline - Delay before surgery (years) & $0.9 \pm 0.7$ & $0.6 \pm 0.9$ & 0.03 \\
\hline - endoscopic surgery (\% patients) & 22.9 & 14.9 & 0.43 \\
\hline - Impression of complete tumor removal (\% patients) & 44.4 & 34.1 & 0.59 \\
\hline - Follow-up (months) & $46.5 \pm 34.5$ & $57.8 \pm 38.3$ & 0.13 \\
\hline
\end{tabular}

Duration of PSMT was not significantly different in cured or not-cured patients, at both evaluations:

3 months: 6.3 months $+/-4.3$ (C) vs 7.5 months $+/-4.2(\mathrm{NC}), \mathrm{p}=0.36$

Long-Term: 5.9 months $+/-4.1$ (C) vs 7.3 months $+/-4(\mathrm{NC}), \mathrm{p}=0.21$
PSMT was more beneficial for patients with somatotroph adenoma larger than $15 \mathrm{~mm}$ :

Group 1: Size $<15 \mathrm{~mm}+$ No PSMT

Group 2: Size $<15 \mathrm{~mm}+$ PSMT

Group 3: Size $>15 \mathrm{~mm}+$ No PSMT

Group 4: Size $>15$ mm+ PSMT

In multivariate analysis, group 3 (Size $>15 \mathrm{~mm}$, No PSMT) had a significant lower remission rate than the 3 other groups $(\mathrm{p}<0.01)$.

Noteworthy, no patient with a more than $18 \mathrm{~mm}$ adenoma or a mean GH exceeding $35 \mathrm{ng} / \mathrm{ml}$ at diagnosis was cured by surgery without PSMT.
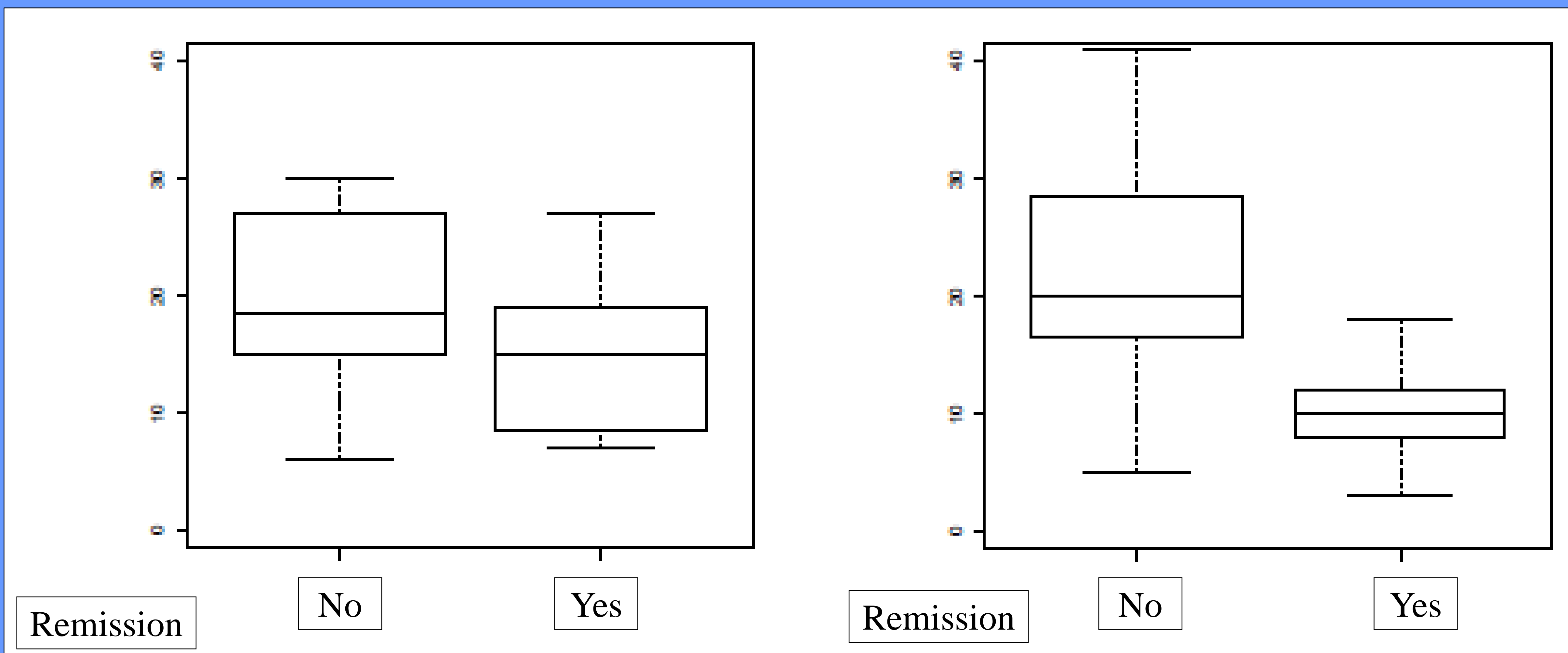

Long Term remission and size of the adenoma in patients with (left) or without (right) PSMT

\section{Conclusion}

Pre-surgical medical treatment (PSMT) significantly improved short and long-term remission in operated acromegalic patients, independently of its duration and main confounding factors, and seemed to be especially interesting in adenomas larger than $15 \mathrm{~mm}$. 